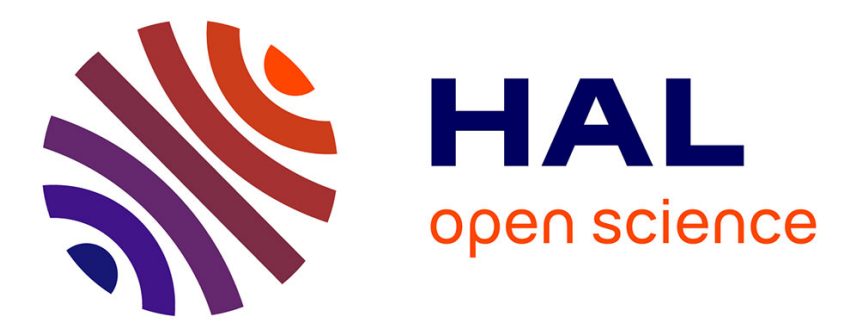

\title{
3D Local Binary Pattern for PET image classification by SVM, Application to early Alzheimer disease diagnosis
}

Christophe Montagne, Andreas Kodewitz, Vincent Vigneron, Virgile Giraud, Sylvie Lelandais

\section{- To cite this version:}

Christophe Montagne, Andreas Kodewitz, Vincent Vigneron, Virgile Giraud, Sylvie Lelandais. 3D Local Binary Pattern for PET image classification by SVM, Application to early Alzheimer disease diagnosis. 6th International Conference on Bio-Inspired Systems and Signal Processing (BIOSIGNALS 2013), Feb 2013, Barcelona, Spain. pp.145-150. hal-00762837

\section{HAL Id: hal-00762837 https://hal.science/hal-00762837}

Submitted on 1 Feb 2014

HAL is a multi-disciplinary open access archive for the deposit and dissemination of scientific research documents, whether they are published or not. The documents may come from teaching and research institutions in France or abroad, or from public or private research centers.
L'archive ouverte pluridisciplinaire HAL, est destinée au dépôt et à la diffusion de documents scientifiques de niveau recherche, publiés ou non, émanant des établissements d'enseignement et de recherche français ou étrangers, des laboratoires publics ou privés. 


\title{
3D Local Binary Pattern for PET image classification by SVM Application to early Alzheimer disease diagnosis
}

\author{
Christophe Montagne, Andreas Kodewitz, Vincent Vigneron, Virgile Giraud and Sylvie Lelandais \\ University of Evry, IBISC Laboratory, 40 rue du Pelvoux, CE 1455, 91020 Evry cedex, FRANCE \\ \{christophe.montagne,andreas.kodewitz,vincent.vigneron,sylvie.lelandais\}@univ-evry.fr
}

\begin{abstract}
Keywords: Local Binary Pattern, Feature Extraction, Positron Emission Tomographic images, Alzheimer disease, Machine Learning

Abstract: The early diagnostic of Alzheimer disease by non-invasive technique becomes a priority to improve the life of patient and his social environment by an adapted medical follow-up. This is a necessity facing the growing number of affected persons and the cost to our society caused by dementia. Computer based analysis of Fluorodeoxyglucose PET scans might become a possibility to make early diagnosis more efficient. Temporal and parietal lobes are the main location of medical findings. We have clues that in PET images these lobes contain more information about Alzheimer's disease. We used a texture operator, the Local Binary Pattern, to include prior information about the localization of changes in the human brain. We use a Support Vector machine (SVM) to classify Alzheimer's disease versus normal control group and to get better classification rates focusing on parietal and temporal lobes.
\end{abstract}

\section{INTRODUCTION}

The number of people affected by the Alzheimer Disease (AD) is growing. In 2010, this dementia affected 35.6 million people (Wimo and Prince, 2010). This disease affects patient himself and his social environment. The detection of the early states of Alzheimer disease allows to begin some treatments for the patient and slow down the progress of the disorder (Salmon, 2008). Most of works focused on temporal lobes, few of them on parietal lobes (Kodewitz et al., 2011). Some works on the SPECT-scans (Single Photon Emission Computed Tomography) are due to Ramìrez with a computer-aided diagnosis based on a selection of image parameters (first and second order statistics) and Support Vector Machine (SVM) (Ramìrez et al., 2009). Others methods with PETscans (Positron Emission Tomography) are based on covariance analysis of voxels to classify $\mathrm{AD}$ versus Normal Control (NC) (Scarmeas et al., 2004). The main idea of this work is that we can automaticaly analyse the PET scans to detect AD patient versus NC patient using textural features. This classification can help doctors to make their early diagnostic and try to localize some brain areas where Alzheimer Disease is growing. In our study, we use 3D-scans extracted from the ADNI database (Alzheimer's Disease Neuroimaging Initiative) and we propose to use a textural operator which is the Local Binary Pattern (LBP) (Pietikäinen and Ojala, 2000) extended to the 3D. Then this new feature was studied by an ANalysis Of VAriance (ANOVA). The analysis highlighted a link between LBP patterns and the type of patient or some brain areas. Based on this new feature, we train a machine learning algorithm SVM to classify $A D$ versus NC PET-scans. For this step, in a first time we use as Region Of Interest (ROI) parietal and temporal lobes, and then, in a second time full PET-scans are considered.

In the first section we describe the materials used in this study. Secondly we introduce the 3D Local Binary Pattern that we developped. Then we explain the ANOVA method and the obtained results. Finally after a short presentation of SVM, we discuss on the results of the classification using the LBP caracteristics and we conclude.

\section{MATERIALS}

In this study, PET-scan provides threedimensional functional imaging data that measures the metabolism in the brain which are acquired by a non-invasive method, Figure 1 shows how the $\mathrm{AD}$ reduces the metabolism in the brain. This scan is obtained by $18 \mathrm{~F}$-fluorodesoxyglucose (in short 
18F-FDG) injection to a patient. This radioactive isotope allows to capture the distribution of brain activity. With this technique, we can detect the first abnormalities before structural alteration (Bennys et al., 2001).

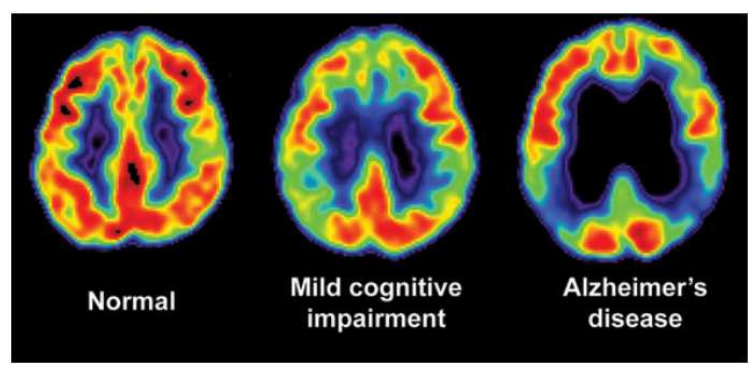

Figure 1: PET scans of AD, MCI and NC patient.

\subsection{ADNI Database}

The American Alzheimer's Disease Neuroimaging Initiative (ADNI, http: / / www . loni.ucla.edu/ $\mathrm{ADNI} /$ ) collects data of patients affected by $\mathrm{AD}$, mild cognitive impairment (MCI) and normal control group (NC). This includes diagnosis based on mental state exams (e.g. mini mental state exam (MMSE)), biomarkers, MRI and PET scans. In this database we chose PET scans with 18F-FDG tracer from AD and NC patients. Each image contains $91 \times 109 \times 91$ voxels after normalization (the PET-scans have initialy different sizes). For our purpose we extracted $1663 \mathrm{D}$-scans (one 3D-scan for one patient) which are sorted in $82 \mathrm{AD}$ and $84 \mathrm{NC}$. Moreover, the AD scans are taken early in the disease to simulate a situation of early diagnosis.

\subsection{Brain Atlas}

Today, the definite diagnosis of AD is based on the post-mortem observation of intracellular neurofibrillary tangles (NTF): $\beta$-amyloid deposition in the form of extracellular senile plaques and blood vessel deposits, synapse dysfunction and loss. NTF deposition originates in the medial temporal lobes and then begins to cluster in the adjacent inferior temporal and posterior cingulate cortex in mild $\mathrm{AD}$, and finally spreads to the parieto-temporal and prefrontal association cortices. Medical doctors use this information in their analysis of PET scans. They search for abnormal variations in the brain metabolism and do their diagnosis based on the location of these abnormalities. In order to emulate this method, we decide to use a brain atlas which gives us a brain model. With the Matlab toolbox WFU PickAtlas (Maldjian et al.,
2003), using the Talairach deamon by Lancaster et al. (Lancaster et al., 2000), it is even possible for nonspecialists to select a volume of interest in the brain by knowing the name of a certain area, e.g. the name of the lobe, and create an indexed mask for this volume of interest. We used these prerequisites to create a simplified map which distinguishes only three zones: temporal lobes, parietal lobes and the rest of the brain (see figure 2). Each voxel of a PET-scan matches with a voxel of this atlas because our atlas size is the same as our normalised brain PET-scans. So we have a unique model of brain for all individuals. Parietal and temporal areas are considered identical from one brain to another and adopt a location and volume average. Parietal lobes contain 26850 voxels, temporal lobes contains 33103 voxels and rest of the brain contain 119907 voxels in each PET-scan.

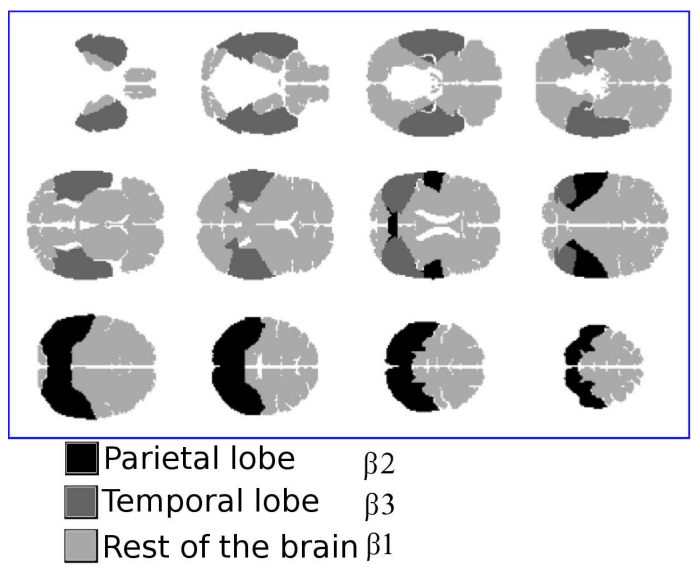

Figure 2: Brain atlas.

\section{LOCAL BINARY PATTERN}

\subsection{D Local Binary Pattern}

LBP was proposed by Pietikäinen et Ojala in the 90's (Pietikäinen and Ojala, 2000). The idea is to give a pattern code to each pixel. Gray level of the central pixel $g_{c}$ is compared to gray level of each neighboring pixel $g_{i}$. Then by thresholding, a value of 0 or 1 is associated to each neighboring pixel.

$$
L B P_{P, R}^{2 d}=\sum_{i=0}^{P-1} s\left(g_{i}-g_{c}\right) 2^{i}, \text { with } s(x)= \begin{cases}1 & \text { if } x \geq 0 \\ 0 & \text { else. }\end{cases}
$$

where $P$ is the number of neighbor pixels separated from $R$ pixel(s) to the central pixel $c$. Figure 3 illustrates this feature. 

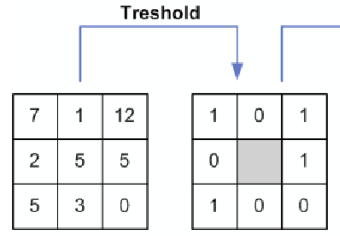

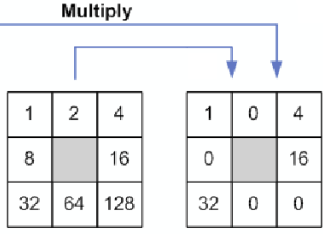

LBP $=1+4+16+32=5$

Figure 3: Example of 2D-LBP with $P=8$ and $R=1$.

\subsection{D Local Binary Pattern}

The main idea of this work is an adaptation of the LBP to capture the 3D structure of texture. We need LBP patterns which have a rotation invariance and qualify the AD PET-scans. Some works have been performed on the extend of the Local Binary Pattern method for characterisation of 3D textures by L. Paulhac (Paulhac et al., 2008). His vision of 3D-LBP consists on a superposition of circles that represent a spherical neighborhood. Each circle can be encoded like a 2DLBP. Our proposition consists to select 6 nearest voxels and to order them for encoding pattern (see figure 4).

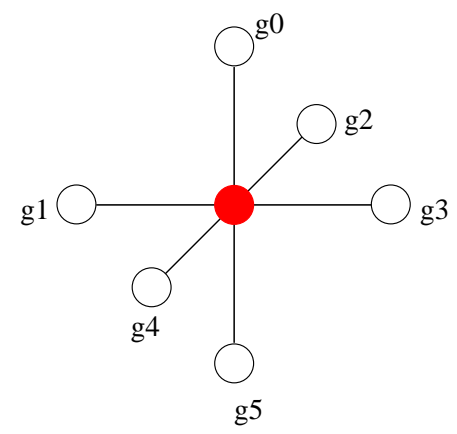

Figure 4: 3D-LBP with 6 neighbors $L B P_{6,1}^{3 d}$.

By this way, we have $2^{6}=64$ possible patterns that we merge in 10 groups according to geometrical similarities (see figure 5). Each group is filled with patterns which have the same numbers of neigbor voxels with a gray level higher than the central voxel $c$. The idea was to keep a rotation invariance in each group. Table 1 defines these groups with:

$$
\operatorname{card}(c)=\sum_{i=0}^{P-1} s\left(g_{i}-g_{c}\right)
$$

where $P=6$ is the number of voxels in neighborhood and $R=1$ or $R=2$ the distance between the central voxel and its neighbors. We use two values of $R$ to capture micro and macrostructure of the texture with our 3D-LBP. In equation 2, $\operatorname{card}(c)$ gives the number of neighbors with an higher gray level than the central voxel.
Table 1: Definition of the 10 groups of patterns rotationally invariant.

\begin{tabular}{c|c|l}
\hline$L B P_{P, R}^{3 d}$ & $\operatorname{card}(c)$ & condition \\
\hline 1 & 0 & \\
2 & 1 & \\
3 & 2 & opposite voxels \\
4 & 2 & bend voxels \\
5 & 3 & voxels on the same plane \\
6 & 3 & voxels on different planes \\
7 & 4 & voxels on the same plane \\
8 & 4 & voxels on different planes \\
9 & 5 & \\
10 & 6 & \\
\hline
\end{tabular}

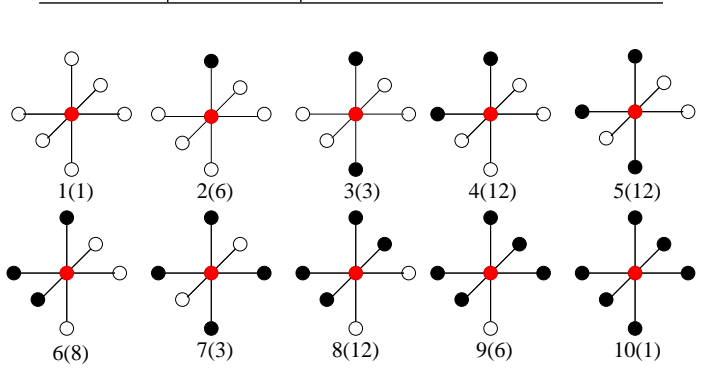

Figure 5: Merging of $2^{6}=64$ patterns in 10 groups. The integer in brackets indicates the number of differents patterns for each group.

In our experimental protocol, the count of these patterns in ROI of PET-scans or in full PET-scans will be the input variable of our machine learning algorithm.

\subsection{ANOVA}

The 3D-LBP features may be an important character for the classification of Alzheimer PET-scan. We performe an analysis of variance to find the link between a variable of interest (the count of 3D-LBP features) and some response variables (type of patient, brain area, type of pattern) which are also called factors and its relevance.

Suppose that the variable to explain $Y$ follows a normal distribution in each population. $y_{i j}$ was a realization of $Y \sim \mathcal{N}\left(\mu_{i}, \sigma\right)$. There are two hypothesis: $H_{0}$-Null hypothesis, every data follows a normal distribution; $H_{1}$-At least one sample follows an other normal distribution.

There are 3 factors:

- $\alpha_{u}$ : type of patient (AD/NC),

- $\beta_{v}$ : brain area (temporal lobe $\beta_{3}$, parietal lobe $\beta_{2}$, rest of brain $\beta_{1}$, full brain $\beta_{0}$ ) (see figure 2 ),

- $\gamma_{w}$ : type of pattern $(1 \ldots 10)$ (see figure 5).

We search the influence of a factor set $\left(\alpha_{u}, \beta_{v}, \gamma_{w}\right)$ on the variable of interest $Y$. This method can be ap- 
plied on each factor or on all together. For example, Eqs. 3 and 4 show a case with a single factor.

$$
y_{i k}=\mu+m_{\alpha_{i}}+\varepsilon_{i k}
$$

where $\mu$ is an offset, $m_{\alpha_{i}}$ the theoretical mean of the sample $i$ and $\varepsilon_{i k}$ are Gaussian i.i.d error terms $\left(\mathcal{N}\left(0, \sigma^{2}\right)\right)$.

$$
\left(\begin{array}{c}
y_{11} \\
\vdots \\
y_{1 n_{1}} \\
y_{21} \\
\vdots \\
y_{2 n_{2}} \\
\vdots
\end{array}\right)=\left(\begin{array}{ccc}
1 & 0 & 0 \\
\vdots & \vdots & \vdots \\
1 & 0 & 0 \\
0 & 1 & 0 \\
\vdots & \vdots & \vdots \\
0 & 1 & 0 \\
\vdots & \vdots & \vdots
\end{array}\right)\left(\begin{array}{c}
m_{\alpha_{1}} \\
m_{\alpha_{2}} \\
m_{\alpha_{3}}
\end{array}\right)+\left(\begin{array}{c}
\varepsilon_{11} \\
\vdots \\
\varepsilon_{1 n_{1}} \\
\varepsilon_{21} \\
\vdots \\
\varepsilon_{2 n_{2}} \\
\vdots
\end{array}\right)
$$

where $m$ is the theoretical mean of the sample. This equation shows the variance decomposition:

$$
\begin{aligned}
& \sum_{i=1}^{3} \sum_{k=1}^{n_{i}}\left(X_{i k}-m\right)^{2}=\sum_{i=1}^{3} n_{i}\left(m_{i}-m\right)^{2}+\sum_{i=1}^{3} \sum_{k=1}^{n_{i}} \varepsilon_{i k}^{2} \\
& S C_{T}^{2}=S C_{A}^{2}+S_{R}^{2}
\end{aligned}
$$

$m_{\alpha_{i}}$ and $m$ are estimated by: $\bar{y}_{i}=\sum_{i=1}^{3} y_{i k}$ and $\bar{y}=$ $\sum_{i=1}^{3} \sum_{k=1}^{n_{i}} y_{i k}$. Under $H_{0}$, we have:

$$
\frac{S C_{T}}{\sigma^{2}} \sim \chi_{n-1}^{2}, \quad \frac{S C_{A}}{\sigma^{2}} \sim \chi_{3-1}^{2}, \quad \frac{S_{R}}{\sigma^{2}} \sim \chi_{n-3}^{2}
$$

and:

$$
T=\frac{S C_{A}^{2} /(3-1)}{S_{R}^{2} /(n-3)} \sim F_{p-1, n-p} .
$$

Under $H_{0}$, the statistics $T$ follows a Fisher distribution with $(3-1, n-3)$ degrees of freedom. The critical value is $T>F_{p-1, n-p, 1-\alpha}$, where $\alpha$ settles the confidence level with which one can reject the hypothesis $H_{0}$ (by convention often chosen to be $95 \%$ ).

Table 2 exposes a part of the results. The $p$-value of each group of pattern on each area of brain is displayed.

The $p$-value is the probability of obtaining a test statistic at least as extreme as the one that was actually observed, assuming that the $H_{0}$ hypothesis is true. The lower is the $p$-value, the higher is the probability of $H_{1}$ to be true. We choose a $p$-value threshold to 0.005 : we accept $H_{1}$ is true with $99.5 \%$ or more (bold $p$-values in the table). We can see $p$-values corresponding to the full brain $\left(\beta_{0}\right)$ or rest of the brain $\left(\beta_{1}\right)$ are higher than our threshold: the count of the 3D-LBP patterns is not informatived in these areas. In the parietal and temporal lobes $\left(\beta_{2}, \beta_{3}\right)$, we notice
Table 2: $p$-values for the different brain areas.

\begin{tabular}{|l|l|ccc|}
\hline \multirow{2}{*}{$\begin{array}{l}\text { LBP Group } \\
\text { number }\end{array}$} & \multicolumn{4}{|c|}{$\operatorname{Pr}(>F)$} \\
\cline { 2 - 5 } & $\beta_{0}$ & $\beta_{1}$ & $\beta_{2}$ & $\beta_{3}$ \\
\hline \hline 1 & $2.13 \mathrm{e}-1$ & $1.42 \mathrm{e}-1$ & $\mathbf{5 . 8 3 e}-\mathbf{5}$ & $2.63 \mathrm{e}-2$ \\
2 & $5.47 \mathrm{e}-1$ & $3.14 \mathrm{e}-1$ & $\mathbf{1 . 6 6 e - 5}$ & $\mathbf{1 . 8 6 e - 4}$ \\
3 & $2.71 \mathrm{e}-1$ & $6.38 \mathrm{e}-1$ & $\mathbf{3 . 1 4 e}-4$ & $\mathbf{2 . 5 1 e}-7$ \\
4 & $7.89 \mathrm{e}-1$ & $1.60 \mathrm{e}-1$ & $\mathbf{1 . 7 6 e - 3}$ & $1.07 \mathrm{e}-2$ \\
5 & $9.77 \mathrm{e}-1$ & $2.24 \mathrm{e}-1$ & $\mathbf{8 . 6 0 e}-5$ & $\mathbf{4 . 6 9 e - 3}$ \\
6 & $7.61 \mathrm{e}-1$ & $4.20 \mathrm{e}-1$ & $\mathbf{1 . 5 6 e - 3}$ & $5.72 \mathrm{e}-3$ \\
7 & $9.22 \mathrm{e}-1$ & $3.38 \mathrm{e}-1$ & $7.40 \mathrm{e}-3$ & $1.39 \mathrm{e}-1$ \\
8 & $7.62 \mathrm{e}-1$ & $4.13 \mathrm{e}-1$ & $\mathbf{2 . 5 0 e}-3$ & $\mathbf{1 . 7 9 e - 3}$ \\
9 & $6.00 \mathrm{e}-1$ & $2.12 \mathrm{e}-2$ & $\mathbf{3 . 2 2 e - 3}$ & $3.69 \mathrm{e}-1$ \\
10 & $3.49 \mathrm{e}-1$ & $2.54 \mathrm{e}-2$ & $1.86 \mathrm{e}-1$ & $6.43 \mathrm{e}-2$ \\
\hline
\end{tabular}

the power of some LBP patterns for discriminating the type of patient $\alpha_{i}$.

These observations allow to use machine learning to create a classifier with the 3D LBP features extracted from the PET-scan.

\section{Classification by Support Vectors Machine}

The SVM was introduced by Vapnik (Vapnik, 1998). We used a description of the SVM which is based on Schölkopf and Smola (Schölkopf and Smola, 2002) works. Consider a training data set consisting of two separable classes in a $n$-dimensional feature space. This means each class forms its own cluster and those clusters do not intersect. From this follows that there exists at least one hyperplane able to separate the two classes with one class on each side of the hyperplane.

We use a Gaussian kernel in SVM, $\gamma \geq$ $0, \quad k\left(x, x^{\prime}\right)=e^{\left(-\left\|x-x^{\prime}\right\|^{2} / \gamma^{2}\right)}$. In figure 6 we can see the contour plot of the error landscape resulting from a grid search on hyperparameter cost $C$ and gamma $\gamma$. $C$ is a cost constant for the Lagrangian. This grid is obtained with the function tune in $\mathrm{R}$. The red circle shows the area with the best parameters.

PET-scans used in this study come from the ADNI database. Each scan contains $91 \times 109 \times 91$ voxels. We selected 82 patients from AD group and 84 from NC group (one patient $=$ one PET-scan). We try to classify AD versus NC. Each voxel in the image is encoded by $L B P_{6,1}^{3 d}$ and $L B P_{6,2}^{3 d}$. We have 10,20 or 30 input variables if we use 1,2 or 3 areas of brain (parietal $\beta_{2}$, temporal $\beta_{3}$, rest of the brain $\beta_{1}$ ) in the classifier. With full brain $\left(\beta_{0}\right)$ we have 10 variables. And when we use the $L B P_{6,1}^{3 d}$ and $L B P_{6,2}^{3 d}$, the number of variables is doubled. 


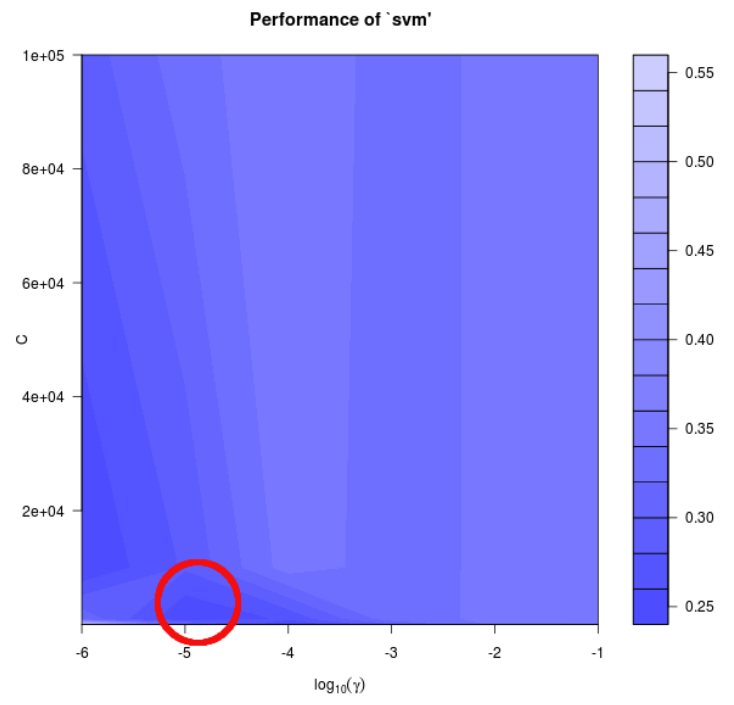

Figure 6: Visualisation of the tunning parameters $C$ and $\gamma$.

These variables constitute our input vector for SVM. In table 3 we see the classification rate for the 3 brain areas or for parietal and temporal lobes. Other results with only one brain area $\left(\beta_{1}, \beta_{2}\right.$ or $\left.\beta_{3}\right)$ or full brain $\left(\beta_{0}\right)$ don't appear in this table because of lower results. The cross validation consists in a number of runs equal to size of the base of a SVM. For each run we divide the main database with a part used for trainning and the other for testing. The mean of all these runs give us the rate of cross validation.

Table 3: Precision, recall and cross validation (CV) for SVM classification - (1): $L B P_{6,1}^{3 d}$, (2): $L B P_{6,2}^{3 d} \cdot \beta_{1,2,3}$ is put for the use of all brain areas; $\beta_{2,3}$ is for temporal and parietal lobes.

\begin{tabular}{|c|c|c|c|c|c|c|}
\hline \multirow{2}{*}{$\begin{array}{l}\text { LBP } \\
\text { type }\end{array}$} & \multirow{2}{*}{$\begin{array}{l}\text { Brain } \\
\text { areas }\end{array}$} & \multicolumn{2}{|c|}{ Precision } & \multicolumn{2}{|c|}{ Recall } & \multirow[b]{2}{*}{ CV } \\
\hline & & $\mathrm{AD}$ & $\mathrm{NC}$ & AD & $\mathrm{NC}$ & \\
\hline \multirow{5}{*}{ (1) } & $\beta_{1}$ & $68 \%$ & $70 \%$ & $70 \%$ & $68 \%$ & $60 \%$ \\
\hline & $\beta_{2}$ & $67 \%$ & $70 \%$ & $70 \%$ & $67 \%$ & $68 \%$ \\
\hline & $\beta_{3}$ & $75 \%$ & $71 \%$ & $67 \%$ & $79 \%$ & $64 \%$ \\
\hline & $\beta_{1,2,3}$ & $89 \%$ & $81 \%$ & $79 \%$ & $90 \%$ & $75 \%$ \\
\hline & $\beta_{2,3}$ & $85 \%$ & $79 \%$ & $76 \%$ & $86 \%$ & $66 \%$ \\
\hline \multirow{5}{*}{ (2) } & $\beta_{1}$ & $64 \%$ & $67 \%$ & $68 \%$ & $63 \%$ & $56 \%$ \\
\hline & $\beta_{2}$ & $73 \%$ & $70 \%$ & $66 \%$ & $76 \%$ & $70 \%$ \\
\hline & $\beta_{2}$ & $65 \%$ & $71 \%$ & $68 \%$ & $76 \%$ & $66 \%$ \\
\hline & $\beta_{1,2,3}$ & $87 \%$ & $79 \%$ & $76 \%$ & $89 \%$ & $75 \%$ \\
\hline & $\beta_{2,3}$ & $82 \%$ & $82 \%$ & $81 \%$ & $83 \%$ & $68 \%$ \\
\hline \multirow{5}{*}{$\begin{array}{l}1 \\
\& \\
(2)\end{array}$} & $\beta_{1}$ & $74 \%$ & $77 \%$ & $77 \%$ & $74 \%$ & $63 \%$ \\
\hline & $\beta_{2}$ & $74 \%$ & $70 \%$ & $67 \%$ & $77 \%$ & $69 \%$ \\
\hline & $\beta_{3}$ & $77 \%$ & $72 \%$ & $67 \%$ & $81 \%$ & $67 \%$ \\
\hline & $\beta_{1,2,3}$ & $87 \%$ & $79 \%$ & $76 \%$ & $89 \%$ & $77 \%$ \\
\hline & $\beta_{2,3}$ & $82 \%$ & $82 \%$ & $81 \%$ & $83 \%$ & $69 \%$ \\
\hline
\end{tabular}

Table 3 shows results obtained with different variables of $L B P_{6,1}^{3 d}$ and $L B P_{6,2}^{3 d}$. We can notice that all the results are very close together. But when we use the 3 brain areas, the SVM classifier reaches better rates than just using parietal and temporal lobes. The best $\mathrm{CV}$ rate is $77 \%$ with $L B P_{6,1}^{3 d} \& L B P_{6,2}^{3 d}$. We can assume that this value is relatively low because the scans correspond to the beginning of the disease when the diagnosis is more difficult than for a patient severely affected.

\section{Conclusion}

We propose a new method to identify patients affected by the Alzheimer Disease from their PET-scan. With the ANOVA we have shown LBP are important features to class AD versus NC PET-scans. Then we train and test a classifier to create an automatic computer-aided diagnosis for the Alzheimer Disease using the LBP caracteristics extracted from the PETscan. We reach to a score of $77 \%$ by using SVM method with $L B P_{6,1}^{3 d}$ and $L B P_{6,2}^{3 d}$ patterns.

Currently we improve this work by slicing in small cubes, in each of these block we count the LBP patterns. Then these counts are used as variables for a Random Forest classification. RF is adapted to compute and to have a good classification rate with this high number of features.

An other way to improve the results can be to develop some new LBP types to capture more accurately the texture of brain in the PET-scans. We can notice that some regions that are outside temporal and parietal lobe contain also a certain amount of discriminating information which is captured with our approach. In this case we can extend the analysed brain areas to find other ROI. A brain mapping of each PETscan can be used to define more cleverly position of the temporal and parietal lobes, or some other subparts. The idea is to customize an atlas for each patient rather than our standart atlas for all the database. This way is already used on IRM-scans. With this, we have to segment brain to find the edges of the different areas. In the same way, merge IRM and PET data will be an interresting search.

\section{Acknowledgements}

Data used in the preparation of this article were obtained from the Alzheimer's Disease Neuroimaging Initiative (ADNI) database (www.loni.ucla.edu/ADNI). As such, the investigators within the ADNI contributed to the 
design and implementation of ADNI and/or provided data but did not participate in analysis or writing of this report. ADNI investigators include (complete listing available at http: //www. loni.ucla.edu/ADNI/Collaboration/ ADNI_Manuscript_Citations.pdf).

\section{REFERENCES}

Alzheimer's disease neuroimaging initiative. http://www.loni.ucla.edu/ADNI/.

Bennys, K., Rondouin, G., Vergnes, C., and Touchon, J. (2001). Diagnostic value of quantitative EEG in alzheimer's disease. Clinical Neurophysiology, 31(3):153-160.

Kodewitz, A., V., V., Montagne, C., and Lelandais, S. (2011). Where to search for alzheimer's disease related changes in pet scans? RITS, Rennes, France.

Lancaster, L., Woldorff, M. G., and Parsons, L. M. (2000). Automated talairach atlas labels for functional brain mapping. Human Brain Mapping, 10:120-131.

Maldjian, J. A., Laurienti, P. J., Burdette, J. H., and Kraft, R. A. (2003). An automated method for neuroanatomic and cytoarchitectonic atlas-based interrogation of fmri data sets. Neurolmage, 19:1233-1239.

Paulhac, L., Makris, P., and Ramel, J. (2008). Comparison between $2 \mathrm{~d}$ and $3 \mathrm{~d}$ local binary pattern methods for characterisation of three-dimensional textures. In Series, B., editor, Lecture Notes in Computer Science, volume 5112/2008, pages 670-679, Pòvoa de Varzim, Portugal. ICIAR.

Pietikäinen, M. and Ojala, T. (2000). rotation-invariant texture classification using feature distributions. Pattern Recognition, 33:43-52.

Ramìrez, J., Gòrriz, J., and et al., D. S.-G. (2009). Computer-aided diagnosis of alzheimer's type dementia combining support vector machines and discriminant set of features. Information Sciences.

Salmon, E. (2008). Différentes facettes de la maladie de type azheimer. Rev Med Liege, 63(5-6):299-302.

Scarmeas, N., Habeck, C. G., and et al., E. Z. (2004). Covariance pet patterns in early alzheimer's disease and subjects with cognitive impairment but no dementia: utility in group discrimination and correlations with functional performance. NeuroImage, 23(1):35 - 45.

Schölkopf, B. and Smola, A. (2002). Learning with Kernels - Support Vector Machines, Regularization, and Beyond. The MIT Press.

Vapnik, V. (1998). Statistical learning theory. Wiley.

Wimo, A. and Prince, M. (2010). World alzheimer report 2010. http://www.alz.co.uk/research/world-report. 